\title{
Deleterious impact of Pseudomonas aeruginosa on cystic fibrosis transmembrane conductance regulator function and rescue in airway epithelial cells
}

\author{
Nguyen Thu Ngan Trinh ${ }^{1,2,5}$, Claudia Bilodeau ${ }^{1,2,5}$, Émilie Maillé ${ }^{1}$, \\ Manon Ruffin ${ }^{1,2}$, Marie-Claude Quintal ${ }^{3}$, Martin-Yvon Desrosiers ${ }^{1}$, \\ Simon Rousseau ${ }^{4}$ and Emmanuelle Brochiero ${ }^{1,2}$
}

Affiliations: ${ }^{1}$ Centre de Recherche du Centre hospitalier de l'Université de Montréal (CRCHUM), Montréal, QC, Canada. ${ }^{2}$ Dept de médecine, Université de Montréal, Montréal, QC, Canada. ${ }^{3}$ Paediatric Otolaryngology Service, Centre Hospitalier Universitaire Sainte-Justine, Montréal, QC, Canada. ${ }^{4}$ Meakins-Christie Laboratories, Dept of Medicine, McGill University, Montréal, QC, Canada. ${ }^{5}$ Both authors contributed equally.

Correspondence: Emmanuelle Brochiero, CRCHUM, Tour Viger, 900 rue St-Denis, Montréal, QC, H2X09A, Canada. E-mail: emmanuelle.brochierodumontreal.ca

ABSTRACT The epithelial response to bacterial airway infection, a common feature of lung diseases such as chronic obstructive pulmonary disease and cystic fibrosis, has been extensively studied. However, its impact on cystic fibrosis transmembrane conductance regulator (CFTR) channel function is not clearly defined. Our aims were, therefore, to evaluate the effect of Pseudomonas aeruginosa on CFTR function and expression in non-cystic fibrosis airway epithelial cells, and to investigate its impact on $\Delta$ F508-CFTR rescue by the VRT-325 corrector in cystic fibrosis cells.

CFTR expression/maturation was evaluated by immunoblotting and its function by short-circuit current measurements.

A 24-h exposure to P. aeruginosa diffusible material (PsaDM) reduced CFTR currents as well as total and membrane protein expression of the wildtype (wt) CFTR protein in CFBE-wt cells. In CFBE- $\Delta$ F508 cells, PsaDM severely reduced CFTR maturation and current rescue induced by VRT-325. We also confirmed a deleterious impact of PsaDM on wt-CFTR currents in non-cystic fibrosis primary airway cells as well as on the rescue of $\triangle$ F508-CFTR function induced by VRT-325 in primary cystic fibrosis cells.

These findings show that CFTR function could be impaired in non-cystic fibrosis patients infected by $P$. aeruginosa. Our data also suggest that CFTR corrector efficiency may be affected by infectious components, which should be taken into account in screening assays of correctors.

$@$ ERSpublications

Exposure of airway epithelial cell to $P$. aeruginosa impairs CFTR function, expression and rescue by correctors http://ow.ly/IleTw

This article has supplementary material available from erj.ersjournals.com

Received: April 232014 | Accepted after revision: Dec 282014 | First published online: March 182015

Support statement: This study was funded by Cystic Fibrosis Canada (research grant E. Brochiero, studentship C. Bilodeau), CRCHUM and Université de Montréal (E. Brochiero and C. Bilodeau), the Canadian Thoracic Society, the Fondation GO and the CORAMH (Studentship N.T.N. Trinh). Funding information for this article has been deposited with FundRef.

Conflict of interest: None declared.

Copyright OERS 2015 


\section{Introduction}

Chloride and bicarbonate secretion through cystic fibrosis transmembrane conductance regulator (CFTR) channels is crucial in airways to maintain an adequate periciliary liquid volume and an efficient mucociliary clearance [1], as well as to control airway surface liquid $\mathrm{pH}$ [2], which are necessary to protect the lung against infections. Despite these mechanisms of defence, lungs are frequently exposed to infection/inflammation, which are common features of several lung pathologies including chronic obstructive pulmonary disease (COPD) and cystic fibrosis (CF). The impact of infection on airway epithelia has been extensively studied and it has been established that the continuous presence of pathogens is associated with oxidative stress and chronic inflammatory responses [3-5]. However, the consequences of exposure to infections on CFTR channel expression and function have not been clearly defined. In fact, contradictory results have been reported depending on the bacterial strain, the duration of exposure to infection, as well as the cellular models tested [6-9]. However, it is crucial to understand the outcomes of microbial infection on CFTR. Indeed, a decrease of CFTR function in airways of infected patients would further reduce defence against infections, thus creating a vicious circle of infections impairing CFTR, and then dysfunctional CFTR favouring infections.

In CF, due to mutations in the Cftr gene, dysfunctional CFTR is associated with reduced periciliary volume and mucus accumulation, favouring bacterial colonisation and chronic inflammation $[5,10]$. The prevalence of respiratory pathogens in CF patients varies as a function of age, but the most frequently found bacteria in adults are Pseudomonas aeruginosa, which gradually colonise CF airways [11]. Therefore, many of the current therapies in $\mathrm{CF}$ are directed against infection as well as towards attenuating the inflammatory response $[12,13]$. In addition, many efforts have recently been put towards the development of new strategies directly targeting the basic CFTR defects rather than CF symptoms [14, 15]. New therapies, adapted to each specific class of mutations, are thus progressively emerging. Among them, small molecules called correctors are directed against class II mutations, including the most frequent mutation in CF patients, a deletion of F508 ( $\triangle$ F508-CFTR) leading to improper folding, endoplasmic reticulum trapping and protein degradation $[14,15]$. Efficient CFTR maturation and delivery to the cell membrane has been reported after treatment with various corrector molecules, including corr-2b, corr-4a, VRT-325, MPB-07, KM1160, C18 and VX-809 [16-25]. However, to the best of our knowledge most high throughput screening assays to identify efficient corrector molecules are performed in the absence of infectious products. Notably, it has been previously reported that $P$. aeruginosa exposure reduced CFTR rescue induced by low temperature [6]. Because microbial infection is a key component of CF lung disease, it would thus be crucial to assess the efficiency of small molecules targeting CFTR defects in the presence of infection.

The aim of this study was first to evaluate the impact of $P$. aeruginosa diffusible material (PsaDM) on CFTR function and expression in non-CF airway epithelial cells. We then assessed the effect of PsaDM on CFTR protein degradation and synthesis as well as membrane expression. Finally, we tested if $P$. aeruginosa exoproducts could affect $\Delta$ F508-CFTR maturation and functional rescue by the CFTR corrector VRT-325 in CF airway epithelial cells.

\section{Materials and methods}

Cell culture

CFBE- $\triangle$ F508 and CFBE-wt cell lines (CFBE41o- parental cells [26] stably transduced, respectively, with $\triangle$ F508-CFTR and with wildtype (wt)-CFTR [27]) were used initially. Primary human airway cells were isolated from tissues obtained from non-CF and CF patients (with class II mutations, including $\Delta$ F508/ $\Delta \mathrm{F} 508$ and $\Delta \mathrm{F} 508 / \mathrm{N} 1303 \mathrm{~K}$, median age 23 years, mean forced expiratory volume in $1 \mathrm{~s}$ of $82 \pm 9 \%$ ) undergoing nasal polypectomy at CHUM (Montreal, QC, Canada) and CHU Sainte-Justine (Montreal, QC, Canada) hospitals [28], according to approved ethical protocols. Written informed consents have been obtained from all participants. Cell isolation and culture conditions are detailed in the online supplementary material.

\section{Preparation of $\mathrm{P}$. aeruginosa diffusible material}

In brief, $P$. aeruginosa mucoid strain PACF508 was isolated from the sputum of a CF patient $(\Delta \mathrm{F} 508 /$ $\Delta$ F508, CHU Sainte-Justine) and was grown in lysogeny broth (LB) medium (Life Technologies, Burlington, ON, Canada) for $72 \mathrm{~h}$ (corresponding to the late stationary phase) [29] at $37^{\circ} \mathrm{C}$ with shaking at $250 \mathrm{rpm}$. The bacterial culture was then centrifuged and the supernatant was filtered successively through $0.8,0.45$ and $0.2 \mu \mathrm{m}$ filters. LB was used as the control condition.

\section{Immunoblotting}

Total proteins were extracted from CFBE cells treated or not with VRT-325 (Cystic Fibrosis Foundation Therapeutic Inc. (CFFT) Bethesda, MA, USA), LB, PsaDM, cycloheximide (CHX) (Sigma-Aldrich, St. Louis, MO, USA) and/or with MG132 (Abmole Bioscience, Kowloon, Honk Hong). For CFTR [28] 
and epidermal growth factor receptor (EGFR) [30] detection, the upper section of the membrane was incubated with the polyclonal anti-CFTR 596 antibody (CFFT) or anti-EGFR antibody (anti-erbB1; Cell Signaling Technology, Danvers, MA, USA); whereas the bottom section was incubated with purified mouse anti- $\beta$-actin monoclonal antibody (Cedarlane Laboratory, Burlington, ON, Canada) to ensure equivalent loading. The expression of CFTR was reported as percentage relative to cells with LB, after normalisation to $\beta$-actin signal.

\section{Biotinylation and pull-down}

CFBE-wt cells, treated with LB or PsaDM, were exposed to sulfo-NHS-SS-biotin (Thermo Fisher Scientific Inc., Waltham, MA, USA) before protein solubilisation in RIPA. The proteins were then absorbed on streptavidin beads, transferred onto a Pierce spin column (Thermo Fisher Scientific Inc.), and then eluted with a 2X sample buffer, before separation on SDS-Page and CFTR detection by immunoblotting.

\section{Immunofluorescence assay}

CFTR proteins were detected by immunofluorescence assay on CFBE cells, seeded on Lab-Tek chamber slides (Thermo Fisher Scientific Inc.) and treated with LB or PsaDM before incubation with anti-CFTR 596 antibody (CFFT) and then Alexa Fluor 488 conjugated anti-mouse antibody (Life Technologies Inc.). Slides were finally counterstained with To-Pro-3 iodide (Life Technologies Inc.) and fluorescent images were captured by a Confocal Leica TCS_SP5 microscope (see supplementary methods for details).

\section{Electrophysiology}

Short-circuit currents $\left(I_{s c}\right.$ ) [30-32] were measured through CFBE and primary airway cells [28] that had been cultured on coated permeant filters until they reached an air-liquid interface and formed a polarised epithelium with high resistance [33,34]. Measurements were performed (see supplementary material for details) on intact monolayers or through apical membranes after permeabilisation of the basolateral side with $7.5 \mu \mathrm{M}$ amphotericin B (Sigma-Aldrich), in the presence of a symmetrical physiological solution or a chloride $\left(\mathrm{Cl}^{-}\right)$gradient, as indicated in figure legends.

\section{CFTR mRNA expression}

Total RNA from CFBE cells treated with PsaDM or LB was purified with TRIzol reagent (Life Technologies Inc.) [28], and CFTR mRNA expression was evaluated by $\mathrm{qPCR}$ as described in the supplementary material. The expression of CFTR was reported as percentage relative to cells treated with LB.

\section{Statistics}

The data are presented as means \pm SE. Groups were compared by paired t-test or non-parametric Wilcoxon test, with Statview (SAS Institute, Cary, NC, USA) and GraphPad (La Jolla, CA, USA) software, respectively. A probability of $\mathrm{p}<0.05$ was considered to be significant.

\section{Results}

Decrease in CFTR function induced by $\mathrm{P}$. aeruginosa exoproducts

We first evaluated the impact of PsaDM on CFTR function in polarised airway epithelial CFBE-wt cell monolayers grown on permeant filters. Cells were treated with either LB (control) or PsaDM for a 24-h period before Isc measurements. Just before mounting in an Ussing chamber, intact cell monolayers were washed and bathed with a symmetrical physiological solution supplemented with amiloride in order to inhibit sodium $\left(\mathrm{Na}^{+}\right)$currents through epithelial sodium channel (ENaC) channels. Forskolin (Fsk), 3-isobutyl-1-methylxanthine (IBMX) and genistein were then applied at the apical membrane to stimulate cAMP-activated $\mathrm{Cl}^{-}$secretion through CFTR channels, which was finally inhibited by the $C_{F T R}$ inh $^{-172}$ (fig. 1a). We observed that the change in $I_{s c}$ current $(\Delta I s c)$ after CFTR activation/potentiation $(\Delta I$ Fsk + IBMX $+\Delta$ Igenistein $)$ was significantly reduced in monolayers treated with PsaDM $\left(0.7 \pm 0.3 \mu \mathrm{A} \cdot \mathrm{cm}^{-2}\right)$ when compared to the LB control $\left(2.0 \pm 0.5 \mu \mathrm{A} \cdot \mathrm{cm}^{-2}, \mathrm{p}<0.02\right)$ (fig. 1c). Similarly, $\mathrm{CFTR}_{\text {inh- }}-172$-sensitive CFTR current $\left(\triangle{ }_{\text {ICFTR }}\right.$ inh $\left.^{-172}\right)$ was also markedly affected by exposure to PsaDM $\left(-1.2 \pm 0.4 \mu \mathrm{A} \cdot \mathrm{cm}^{-2}\right.$ compared to $-2.8 \pm 0.6 \mu \mathrm{A} \cdot \mathrm{cm}^{-2}$ for $\mathrm{LB}, \mathrm{p}<0.02$ ) (fig. 1c).

To assess the specific effect of 24-h treatment with PsaDM on $\mathrm{Cl}^{-}$currents through the apical membrane, a series of experiments was performed on CFBE-wt cell monolayers after permeabilisation of the basolateral membrane and establishment of a $\mathrm{Cl}^{-}$gradient. Although cAMP-stimulated and $\mathrm{CFTR}_{\text {inh }}-172$-sensitive $\mathrm{Cl}^{-}$ currents were higher in these conditions (fig. 1d) than through intact monolayers (fig. 1a), a significant reduction was observed in PsaDM treated monolayers (fig. 1f).

We also noted a significant inhibition by PsaDM of UTP-stimulated currents, probably mediated by CaCC channels (11.1 \pm 1.9 and $5.5 \pm 0.8 \mu \mathrm{A} \cdot \mathrm{cm}^{-2}$, in LB and PsaDM treated CFBE-wt cell monolayers, $\left.\mathrm{n}=6, \mathrm{p}<0.04\right)$. 

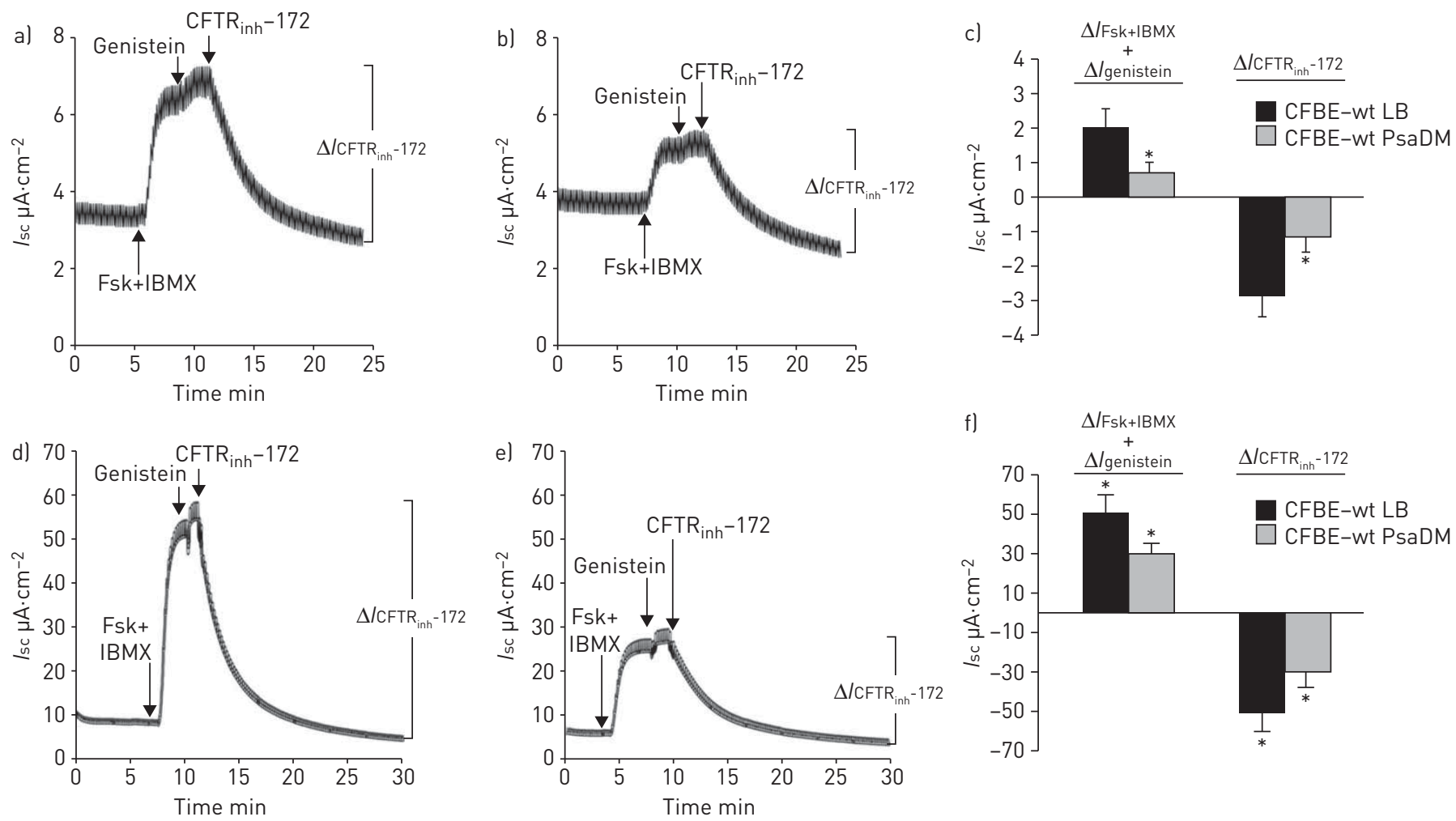

FIGURE 1 Decrease of cystic fibrosis transmembrane conductance regulator (CFTR) function after Pseudomonas aeruginosa diffusible material (PsaDM) treatment in CFBE-wt airway cell monolayers. CFBE-wt cells were cultured on permeant filters at the air-liquid interface and treated for $24 \mathrm{~h}$ with either $10 \%$ of lysogeny broth (LB) medium (a and d) or P. aeruginosa filtrate (PsaDM) (b and e) before short-circuit current (Isc) measurements in an Ussing chamber. a and b) Intact cell monolayers were bathed in a symmetrical physiological medium supplemented with $10 \mu \mathrm{M}$ amiloride and then sequentially exposed to a combination of $10 \mu \mathrm{M}$ forskolin (Fsk) and $100 \mu \mathrm{M}$ 3-isobutyl-1-methylxanthine (IBMX), followed by $30 \mu \mathrm{M}$ genistein and, finally, $20 \mu \mathrm{M}$ CFTR $\mathrm{inh}^{-172 .} \mathrm{d}$ and e) A chloride $\left(\mathrm{Cl}^{-}\right)$gradient was established by bathing the cell monolayers with an asymmetrical physiological solution (low $\mathrm{Cl}^{-}$concentration solution at the apical side and high $\mathrm{Cl}^{-}$concentration at the basolateral side) and the basolateral membrane was permeabilised with $7.5 \mu \mathrm{M}$ amphotericin $\mathrm{B}$. Cells were then sequentially exposed to a combination of Fsk, IBMX and genistein, followed by CFTR inh $_{\text {- }} 172$. Representative Isc traces are reported for each condition, i.e. intact condition and $\mathrm{Cl}^{-}$gradient, for $\mathrm{LB}$ a) and d), respectively, and for PsaDM b) and e), respectively. While quantification of the mean $\Delta I$ Fsk+IBMX+genistein and $\Delta$ ICFTR $_{\text {inh }}-172 I_{\text {sc }}$ after LB and PsaDM exposure is reported for c) intact condition and $\mathrm{f}$ ) $\mathrm{Cl}^{-}$gradient. For intact condition $\mathrm{n}=5$, $\mathrm{p}<0.02$ and for $\mathrm{Cl}^{-}$gradient $\mathrm{n}=6, \mathrm{p}<0.006{ }^{*}: \mathrm{p}<0.05$.

It has to be noted that exposure to PsaDM did not elicit any decrease in transepithelial resistance (1018 \pm 39 and $1398 \pm 97 \Omega \cdot \mathrm{cm}^{2}$ in LB and PsaDM conditions, respectively).

\section{Decrease in CFTR expression induced by $\mathrm{P}$. aeruginosa exoproducts}

We then investigated if the observed decrease in CFTR function induced by $P$. aeruginosa exoproducts in CFBE cells was associated with reduced CFTR expression. We first tested the impact of PsaDM on CFTR mRNA expression in non-CF CFBE-wt cells as well as in CF CFBE- $\Delta$ F508 cells. As shown in figure 2a, the number of CFTR mRNA copies measured by real-time PCR in CFBE-wt cells remains unchanged in the presence of PsaDM. Meanwhile, PsaDM slightly decreased the level of expression of CFTR mRNA in CFBE- $\Delta$ F508 ( $\mathrm{p}<0.006)$ (fig. 2b).

We then assessed the impact of PsaDM on total CFTR-protein expression by immunoblotting. As expected, immature and mature CFTR bands (band B and C, respectively) were detected in CFBE-wt cells (fig. 2c) while the immature band $\mathrm{B}$ was visible in lysates from CFBE- $\Delta$ F508 cells expressing the $\triangle$ F508-CFTR mutant protein (fig. 2d). It was then observed that the intensity of both bands in CFBE-wt was severely reduced in the presence of PsaDM (by $73 \%$ and $40 \%$, respectively, $\mathrm{p}<0.04$ ), when compared to LB control (fig. 2e). In CFBE- $\triangle$ F508 cells, the immature form of CFTR was also affected by PsaDM exposure ( $57 \%$ decrease, $\mathrm{p}<0.0002$ ) (fig. $2 \mathrm{f}$ ). It has to be noted that equal amounts of proteins were loaded in each condition and signals were normalised to $\beta$-actin (which was not altered by PsaDM, see $\beta$-actin detection from the same immunoblots, fig. $2 c$ and $d$ ).

The effect of PsaDM on CFTR membrane expression in CFBE-wt cells was finally evaluated. Immunoblotting assays, after surface biotinylation, revealed that infectious products significantly reduced the level of CFTR plasma-membrane expression compared to the control (fig. $3 \mathrm{a}$ and b). Complementary confocal 

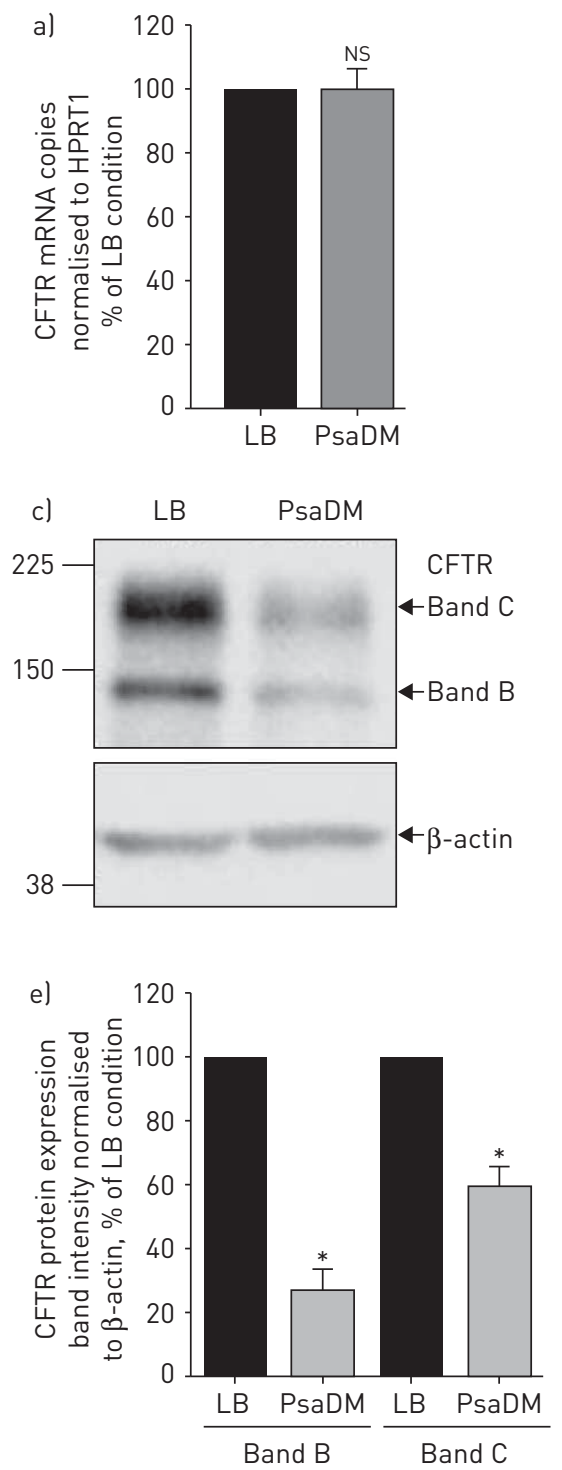
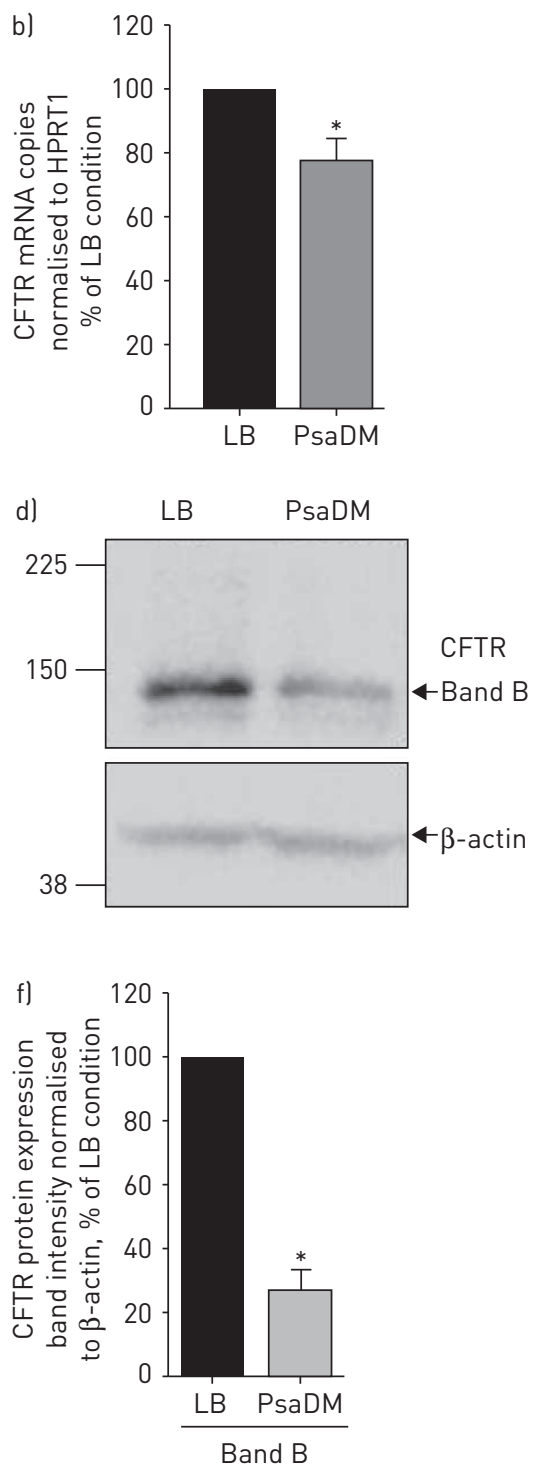

FIGURE 2 Decrease cystic fibrosis transmembrane conductance regulator (CFTR) expression after Pseudomonas aeruginosa diffusible material (PsaDM) treatment. a, $\mathrm{c}$ and e) CFBE-wt or b, d and f) CFBE- $\Delta$ F508 cells were cultured for 8 days (until confluence was reached), then treated with either $10 \%$ of lysogeny broth (LB) medium or PsaDM for $24 \mathrm{~h}$. Levels of CFTR mRNA a) CFBE-wt $(\mathrm{n}=8)$ and b) CFBE- $\Delta$ F508 $(\mathrm{n}=5)$ and protein expression for e) CFBE-wt $(n=5)$ and $f)$ CFBE- $\triangle$ F508 $(n=5)$ were quantified and reported as percentage of the LB control $(p<0.04)$. Representative immunoblots are presented in c) and d) for CFBE-wt and CFBE- $\Delta$ F508, respectively. Ns: nonsignificant; ${ }^{*}: \mathrm{p}<0.05$.

immunofluorescence experiments also showed that CFTR membrane localisation observed in CFBE-wt cells in control conditions (i.e. with LB) was dramatically affected after exposure to PsaDM (fig. 3c). Taken together, these results demonstrated that bacterial exoproducts affected CFTR expression, localisation and function.

\section{Impact of $\mathrm{P}$. aeruginosa exoproducts on CFTR protein degradation and synthesis}

In order to define if the observed decrease in total CFTR protein level in the presence of PsaDM could be due to enhanced protein degradation, we blocked protein translation by exposing CFBE-wt cells to CHX for $0,2,8,18,24$ and $32 \mathrm{~h}$, in the presence or absence of PsaDM. We then measured the remaining level of CFTR protein by immunoblotting (normalised to $\beta$-actin, and expressed as $\%$ of time $0 \mathrm{~h}$ in control condition). As shown in figure $4 \mathrm{a}$, the decrease in CFTR protein abundance following 8, 18 and $32 \mathrm{~h} \mathrm{CHX}$ treatments was slightly, but significantly, higher in the presence of PsaDM $(p<0.05)$. However, this potential increase in CFTR degradation after exposure to PsaDM may not be sufficient to explain the large decrease in CFTR protein level shown in figure 2e. A possible impact of PsaDM on CFTR synthesis was thus assessed by exposing CFBE-wt cells to MG132, a proteasomal inhibitor, for $0,2,8$ and $18 \mathrm{~h}$ in the 
a)

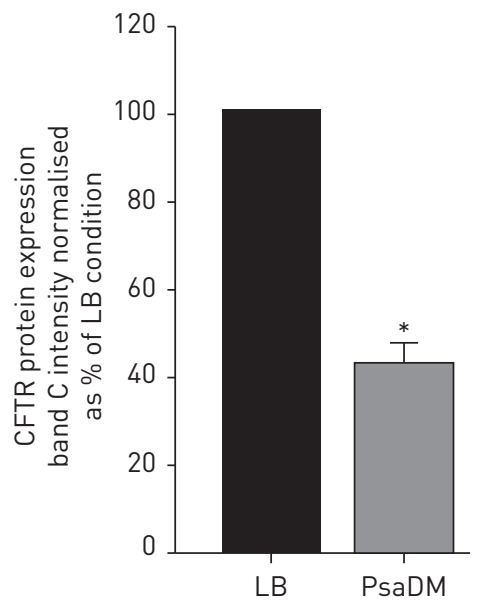

b)

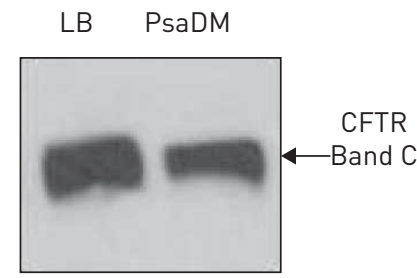

c)

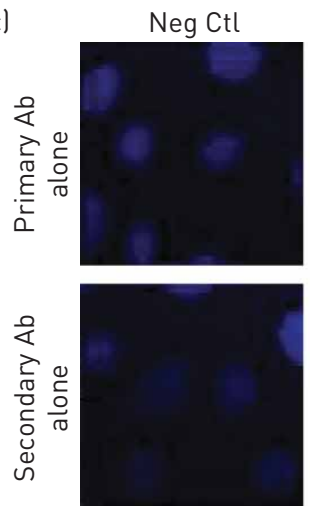

LB

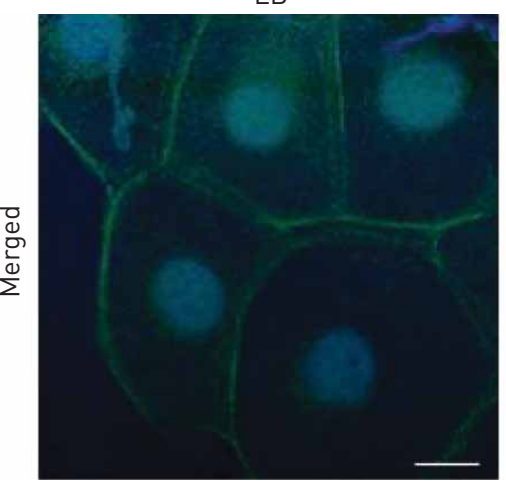

PsaDM

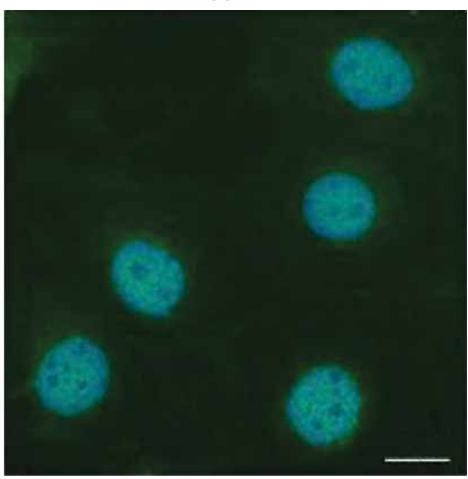

FIGURE 3 Decrease of cystic fibrosis transmembrane conductance regulator (CFTR) expression after Pseudomonas aeruginosa diffusible material (PsaDM) treatment in CFBE-wt airway cells. a and b) The level of CFTR expression at the cell surface in CFBE-wt cells treated for $24 \mathrm{~h}$ with lysogeny broth (LB) medium or PsaDM was measured by immunoblotting after surface biotinylation and reported as a percentage of $L B$ control $(n=6, p<0.04)$. b) A representative immunoblot. c) Subconfluent CFBE-wt cells were treated with either LB or PsaDM for $24 \mathrm{~h}$, then fixed and stained with the anti-CFTR antibodies $(\mathrm{Ab})$ coupled to Alexa Fluor 488 conjugated anti-mouse antibody (green). Nuclei were stained with To-Pro-3 in all conditions. Primary and secondary Ab were also incubated alone for negative controls. Fluorescent images were captured at $\times 100$ magnification by a Confocal Leica TCS_SP5 microscope. Representative images from six different experiments are reported. Neg Ctl: negative control. Scale bar $=10 \mu \mathrm{m}$

presence or absence of PsaDM and then measuring the accumulation of CFTR protein by immunoblotting. We observed (fig. $4 \mathrm{~b}$ ) that the accumulation of wt-CFTR protein bands B and C was higher under control conditions $(\mathrm{LB})$ than in the presence of PsaDM $(\mathrm{p}<0.04)$, suggesting that $P$. aeruginosa exoproducts could reduce CFTR protein synthesis. To confirm that the huge accumulation of CFTR protein observed after proteasomal inhibition with MG132 (MG132+LB at $18 \mathrm{~h}$ ) (fig. 5a and b) was secondary to newly synthesised CFTR proteins, we verified that a co-treatment with CHX (MG132+CHX+LB) totally prevented CFTR accumulation. Again, our data showed that CFTR protein accumulation was severely impacted by PsaDM (MG132+PsaDM) compared to MG132+LB condition $(\mathrm{p}<0.05)$. We also measured the levels of EGFR expression to define if PsaDM could also affect a non-channel membrane protein. Although EGFR accumulation after proteasomal inhibition with MG132 was lower than that of CFTR, we noted that PsaDM exposure did not significantly affected EGFR levels (fig. 5c).

Impaired CFTR rescue in the presence of $\mathrm{P}$. aeruginosa exoproducts

In the light of this evidence pointing towards a deleterious effect of PsaDM on CFTR function and expression, we then decided to investigate if PsaDM had an impact on the ability of the CFTR corrector VRT-325 to rescue $\Delta$ F508-CFTR maturation and function in CF cells. As previously reported [28], we first confirmed that VRT-325 partially rescued CFTR mature band C protein expression (fig. $6 \mathrm{a}$ and b, of note is the comparison between LB and LB+VRT-325) as well as CFTR currents through the apical membrane of CFBE- $\Delta$ F508 cell monolayers (fig. $6 c$ and d, CFBE- $\Delta$ F508 LB versus CFBE- $\Delta$ F508 LB+VRT-325). Indeed, Isc measurements revealed that CFBE- $\Delta$ F508 cell monolayers treated for $24 \mathrm{~h}$ with VRT-325 

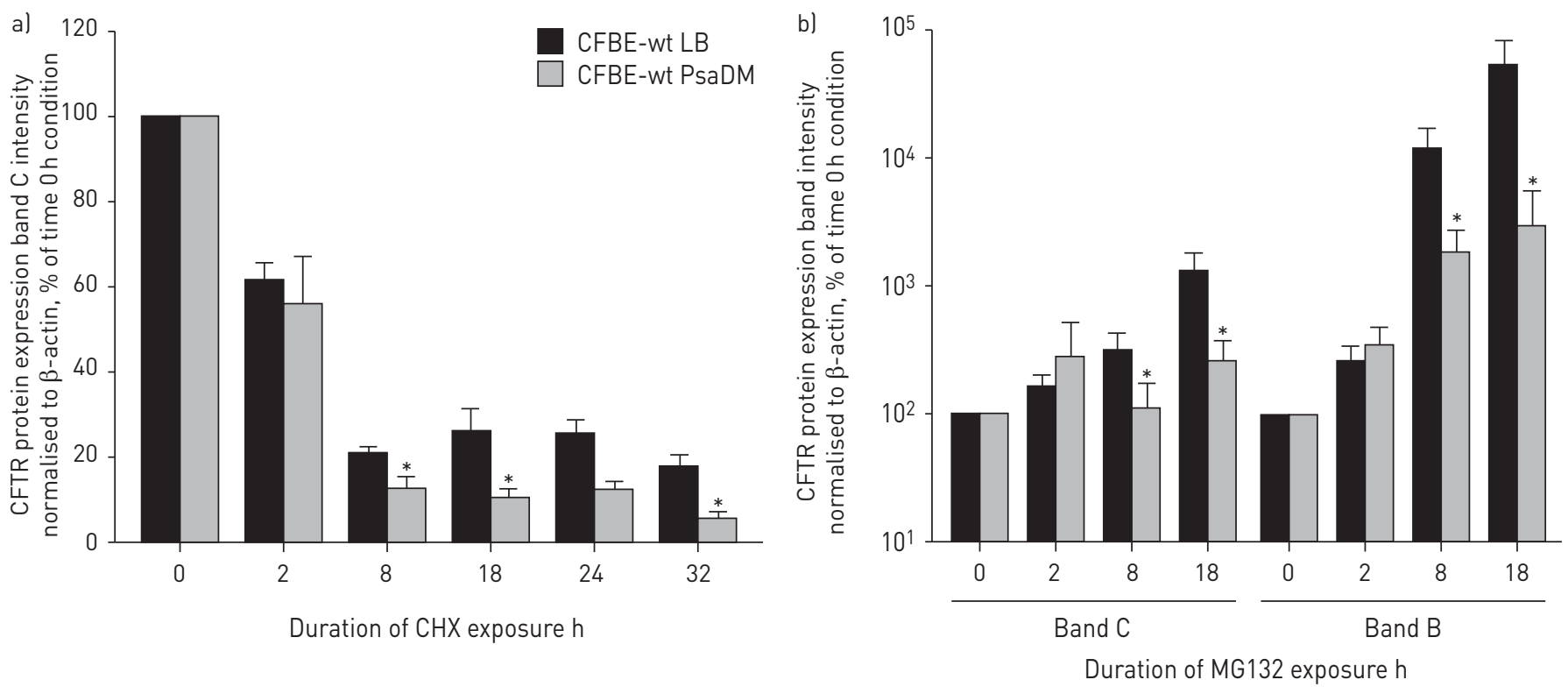

FIGURE 4 Impact of Pseudomonas aeruginosa diffusible material (PsaDM) treatment on the degradation and synthesis of cystic fibrosis transmembrane conductance regulator (CFTR) protein in CFBE-wt airway cell monolayers. CFBE-wt cells were treated with a) $100 \mu \mathrm{g} \cdot \mathrm{mL}^{-1} \mathrm{of}$ cycloheximide $(\mathrm{CHX})$ for 2 , 8 , 18,24 and $32 \mathrm{~h},(\mathrm{n}=6, \mathrm{p}<0.05)$ or with b) $5 \mu \mathrm{M}$ of MG132 for 2,8 and $18 \mathrm{~h}(\mathrm{n}=11, \mathrm{p}<0.04)$, in the presence of $10 \%$ lysogeny broth (LB) medium or PsaDM. The whole cell lysates were subjected to SDS-PAGE separation and analysed by immunoblotting with anti- $\beta$-actin (used as loading control) and anti-CFTR antibodies to evaluate the kinetics of CFTR protein degradation in the presence of CHX (a) and the accumulation of newly synthesised CFTR proteins in the presence of the proteasomal inhibitor MG132 (b). *: $\mathrm{p}<0.05$.

elicited significantly higher cAMP-activated $\mathrm{Cl}^{-}$currents, sensitive to the $\mathrm{CFTR}_{\mathrm{inh}}-172$, compared to the LB control condition (fig. $6 c$, d and f).

However, in the presence of PsaDM for $24 \mathrm{~h}$ (PsaDM+VRT-325) we noted a drastic decrease in band C maturation/expression, compared to the signal in the LB+VRT-325 ( $\mathrm{p}<0.02)$ (fig. 6a and b). The outcome of PsaDM on the rescue of $\triangle$ F508-CFTR channel function by VRT- 325 was then assessed. As shown in figure 6d-f, cAMP-activated $\mathrm{Cl}^{-}$secretion following CFTR activation/potentiation $(\triangle I$ Fsk+IBMX+genistein) was notably decreased in PsaDM+VRT-325 $\left(0.66 \pm 0.68 \mu \mathrm{A} \cdot \mathrm{cm}^{-2}\right)$ when compared to LB+VRT-325 $\left(2.16 \pm 0.32 \mu \mathrm{A} \cdot \mathrm{cm}^{-2}, \mathrm{p}<0.03\right)$. Furthermore, VRT-325-rescued $\mathrm{CFTR}_{\text {inh }^{-172}}$-sensitive CFTR currents in the presence of PsaDM (PsaDM+VRT-325, $-1.74 \pm 0.58 \mu \mathrm{A} \cdot \mathrm{m}^{-2}$ ) were also smaller than in LB (LB+VRT-325, $-2.89 \pm 0.47 \mu \mathrm{A} \cdot \mathrm{cm}^{-2}, \mathrm{p}<0.02$ ) (fig. $6 \mathrm{~d}-\mathrm{f}$ ). These results indicated that the rescue of $\Delta \mathrm{F} 508$-CFTR channel function by the CFTR corrector VRT-325 is strongly impaired by P. aeruginosa exoproducts.

\section{Deleterious impact of $\mathrm{P}$. aeruginosa exoproducts on CFTR function in primary human airway epithelial cells}

The effect of PsaDM on CFTR function was finally assessed in primary human airway epithelial cells isolated from non-CF and CF patients. In non-CF airway cells, we confirmed a significant reduction in cAMP-activated $\mathrm{Cl}^{-}$secretion ( $\triangle$ IFsk+IBMX of $13.9 \pm 3.5$ versus $8.9 \pm 2.7 \mu \mathrm{A} \cdot \mathrm{cm}^{-2}$, respectively under $\mathrm{LB}$ and PsaDM conditions, $\mathrm{p}<0.05)$, as well as $\mathrm{CFTR}_{\mathrm{inh}}-172$-sensitive $\left(\Delta \mathrm{ICFTR}_{\mathrm{mh}}-172,-18.5 \pm 3.1 \mu \mathrm{A} \cdot \mathrm{cm}^{-2}\right.$ in $\mathrm{LB}$ versus $-13.5 \pm 3.1 \mu \mathrm{A} \cdot \mathrm{cm}^{-2}$ in PsaDM) CFTR currents by P. aeruginosa exoproducts (fig. 7). Moreover, the rescue of CFTR currents in CF airway primary cells, induced by VRT-325, was also impaired in the presence of PsaDM ( $\Delta$ IFsk+IBMX of $1.1 \pm 0.4 \mu \mathrm{A} \cdot \mathrm{cm}^{-2}$ versus $0.4 \pm 0.4 \mu \mathrm{A} \cdot \mathrm{cm}^{-2}$, respectively in the presence of

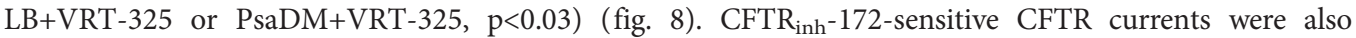
slightly reduced in the presence of PsaDM.

\section{Discussion}

Altogether, our data demonstrated that exoproducts from P. aeruginosa impacted CFTR function in both CFBE-wt cells and primary human airway epithelial cells isolated from non-CF patients. A decrease in CFTR protein expression was observed, which could be due, at least in part, to enhanced protein degradation and reduced protein synthesis. P. aeruginosa exoproducts also affected CFTR membrane expression. Importantly, our study also revealed that the functional rescue of $\Delta$ F508-CFTR by a CFTR corrector in CFBE- $\triangle$ F508 cells and CF primary human airway epithelial cells was impaired in the presence of bacterial products. 
a)
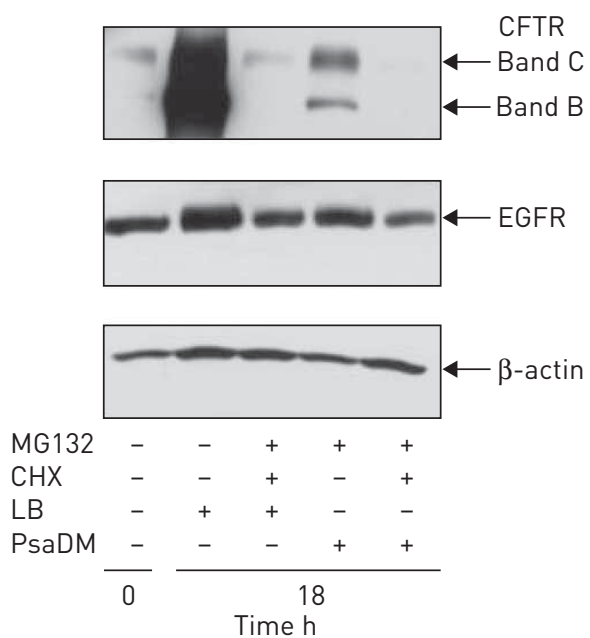

c)

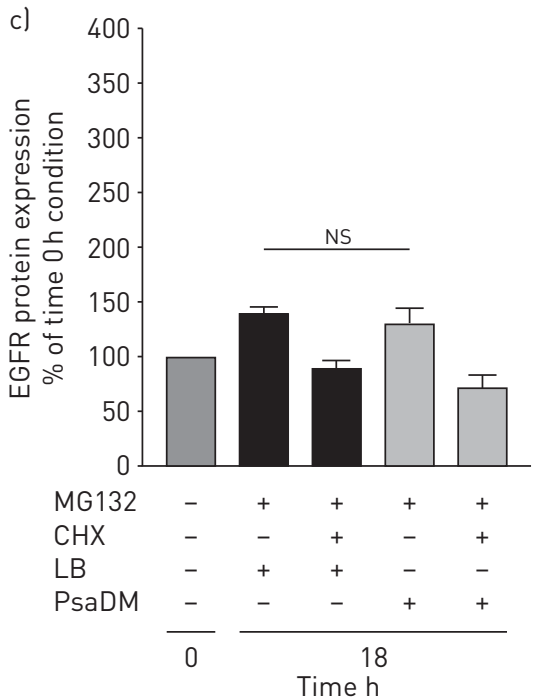

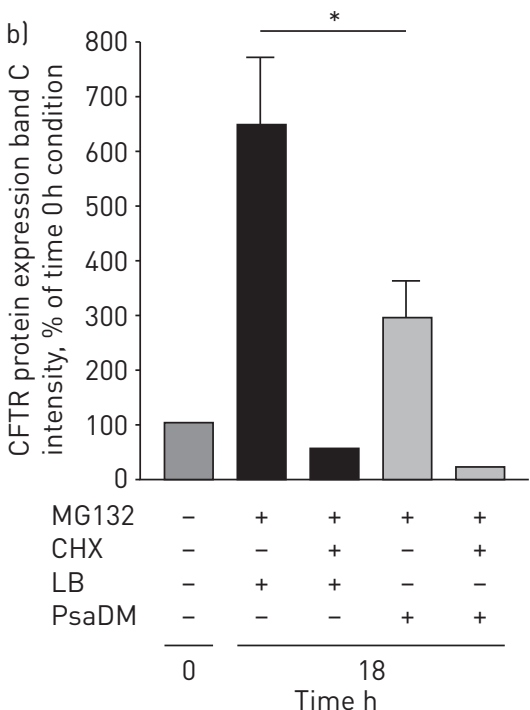

d)

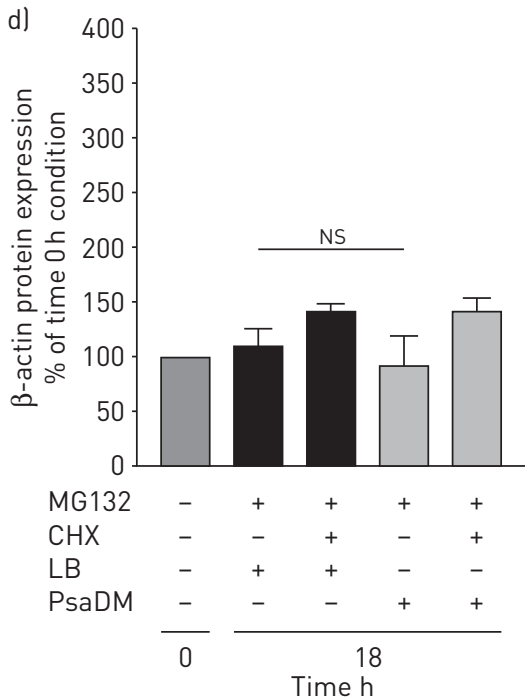

FIGURE 5 Impact of Pseudomonas aeruginosa diffusible material (PsaDM) treatment on cystic fibrosis transmembrane conductance regulator (CFTR) and epidermal growth factor receptor (EGFR) proteins in CFBE-wt airway cell monolayers. CFBE-wt cells were treated with $5 \mu \mathrm{M}$ of MG132 (for $18 \mathrm{~h}$ ) alone or in combination with cycloheximide (CHX) (MG132 $+\mathrm{CHX}$ ), in the presence of lysogeny broth (LB) medium or PsaDM. a and b) CFTR, a and c), EGFR, and a and d) $\beta$-actin proteins were detected by immunoblotting with their respective antibodies. A representative immunoblot is presented in a) and protein levels were quantified and reported as a percentage of the LB control in $b-d$ ). Ns: nonsignificant; $n=6 ; p<0.03$; ${ }^{*}: \mathrm{p}<0.05$.

\section{Impaired CFTR function by infectious products}

Our Ussing chamber experiments on CFBE-wt cells presented in figure 1 demonstrated that a 24-h exposure to diffusible materials from a clinical isolate of $P$. aeruginosa (PACF508 strain) induced a significant reduction in cAMP-activated $\mathrm{Cl}^{-}$currents through CFTR channels. We are aware that the observed decrease in cAMP-activated currents after exposure to PsaDM could be due to a secondary effect on other cAMP-dependent channels, such as the basolateral KvLQT1 potassium channel. To further define if the effect of PsaDM is specific of CFTR, another series of experiments were undertaken on CFBE-wt cells, after permeabilisation of the basolateral membrane and establishment of a $\mathrm{Cl}^{-}$gradient, to focus on $\mathrm{Cl}^{-}$currents through the apical membrane. As presented in figure $1 \mathrm{~d}-\mathrm{f}$, a significant reduction in both cAMP-activated and CFTR $_{\text {inh }}$-172-sensitive currents was observed in PsaDM treated CFBE-wt cell monolayers. This result confirmed the impact of $P$. aeruginosa exoproducts on CFTR function. It has to be noted that PsaDM also reduced UTP-activated currents through CaCC channels, thus potentially precluding a possible compensatory effect of the CaCC after inhibition of CFTR channels in infectious conditions. 


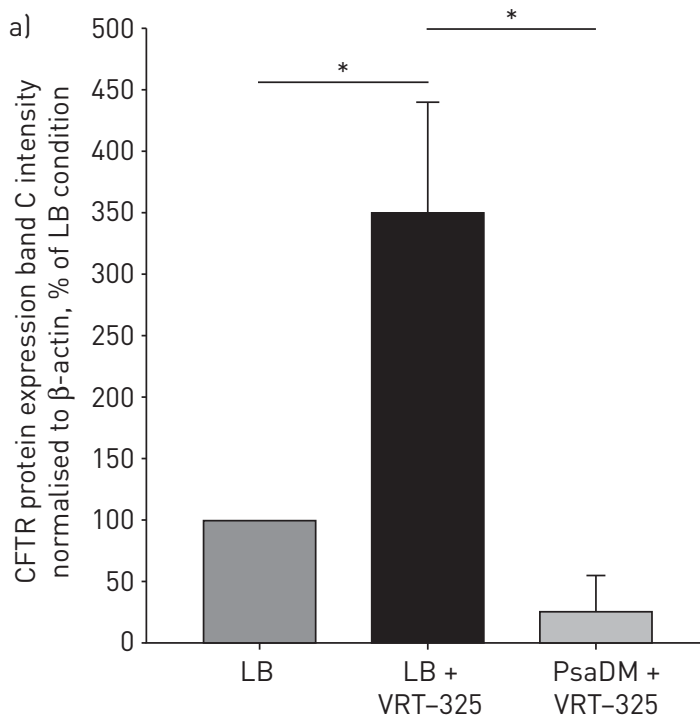

b) VRT-325 -
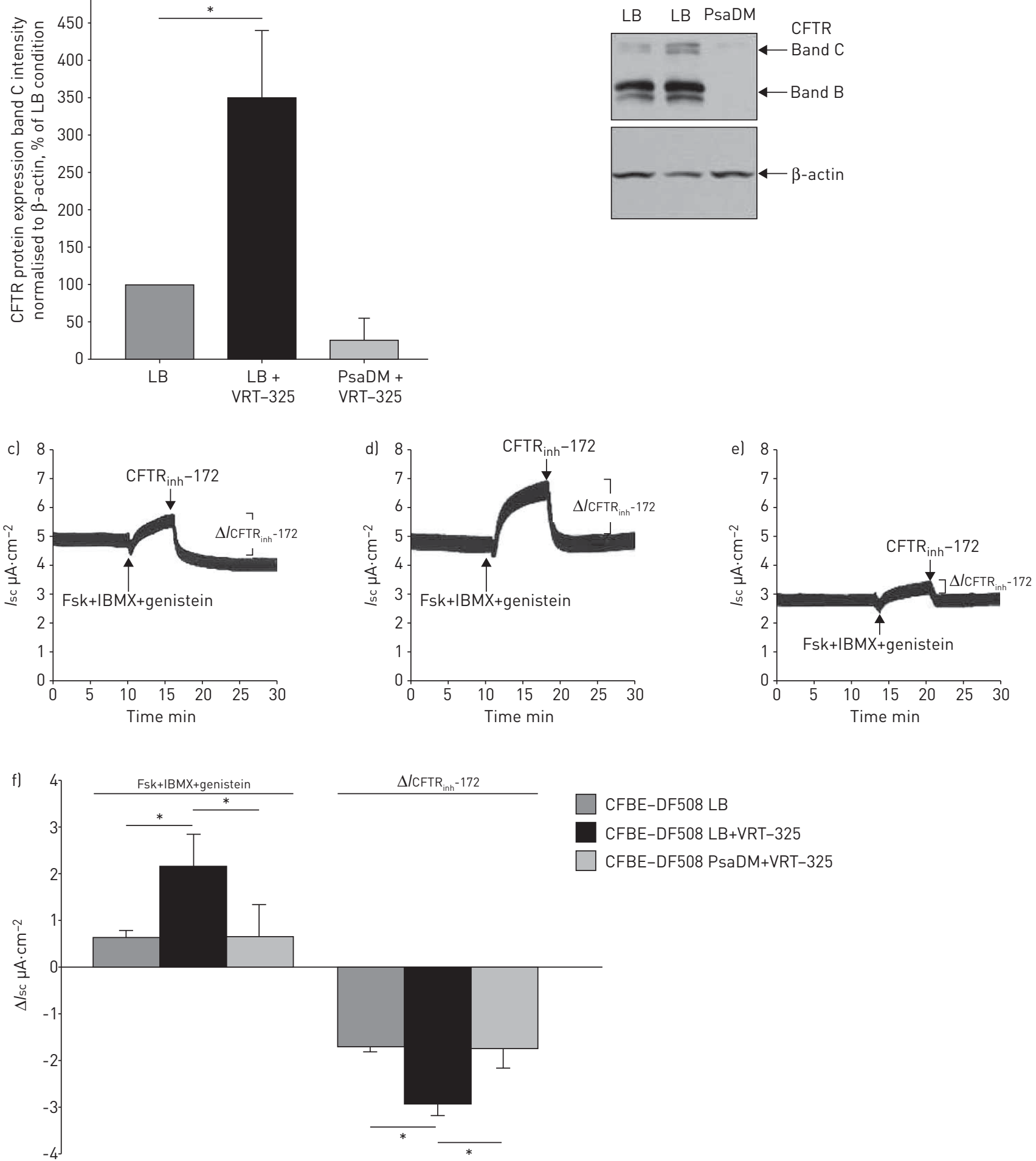

CFBE-DF508 LB

CFBE-DF508 LB+VRT-325

CFBE-DF508 PsaDM+VRT-325

FIGURE 6 Impairment of $\Delta$ F508 cystic fibrosis transmembrane conductance regulator ( $\triangle$ F508-CFTR) functional rescue after Pseudomonas aeruginosa diffusible material (PsaDM) treament. a) CFTR band C protein expression in nontreated CFBE- $\Delta$ F508 cells (i.e. lysogeny broth (LB) medium alone) and in CFBE- $\Delta$ F508 cells treated with $5 \mu \mathrm{M}$ VRT-325 for $24 \mathrm{~h}$ in the presence of $10 \% \mathrm{LB}$ (LB+VRT-325) or PsaDM (PsaDM+VRT-325) was quantified and reported as percentage of the LB control $(\mathrm{n}=8, \mathrm{p}<0.04)$. b) A representative immunoblot. $\mathrm{c}-\mathrm{f})$ Short-circuit currents $\left(I_{\mathrm{sc}}\right)$ were measured in an Ussing chamber through CFBE- $\Delta$ F508 cells, cultured on permeant filters at the air-liquid interface. After mounting in an Ussing chamber, cells were bathed with an asymmetrical physiological solution (low chloride $\left(\mathrm{Cl}^{-}\right)$concentration solution at the apical side and high $\mathrm{Cl}^{-}$concentration at the basolateral side) and the basolateral side was permeabilised with $7.5 \mu \mathrm{M}$ amphotericin B. Upon Isc stabilisation, cells were exposed to a mix of forskolin (Fsk), 3-isobutyl-1-methylxanthine (IBMX) and genistein, and then with $\mathrm{CFTR}_{\text {inh }}$ 172. Representative Isc traces are reported for each condition: c) nontreated (LB), d) treated for $24 \mathrm{~h}$ before electrophysiological measurements with VRT-325 or e) treated with a combination of VRT-325 and PsaDM. f) Quantifications of the mean $\Delta I$ Fsk+IBMX+genistein and $\Delta$ ICFTR $_{\text {inh }}-172 I_{\text {sc }}$ in LB, LB+VRT-325 and PsaDM+VRT-325 conditions $(\mathrm{n}=6, \mathrm{p}<0.03) . \mathrm{n}=6$; ${ }^{*}$ : $<<0.05$. 

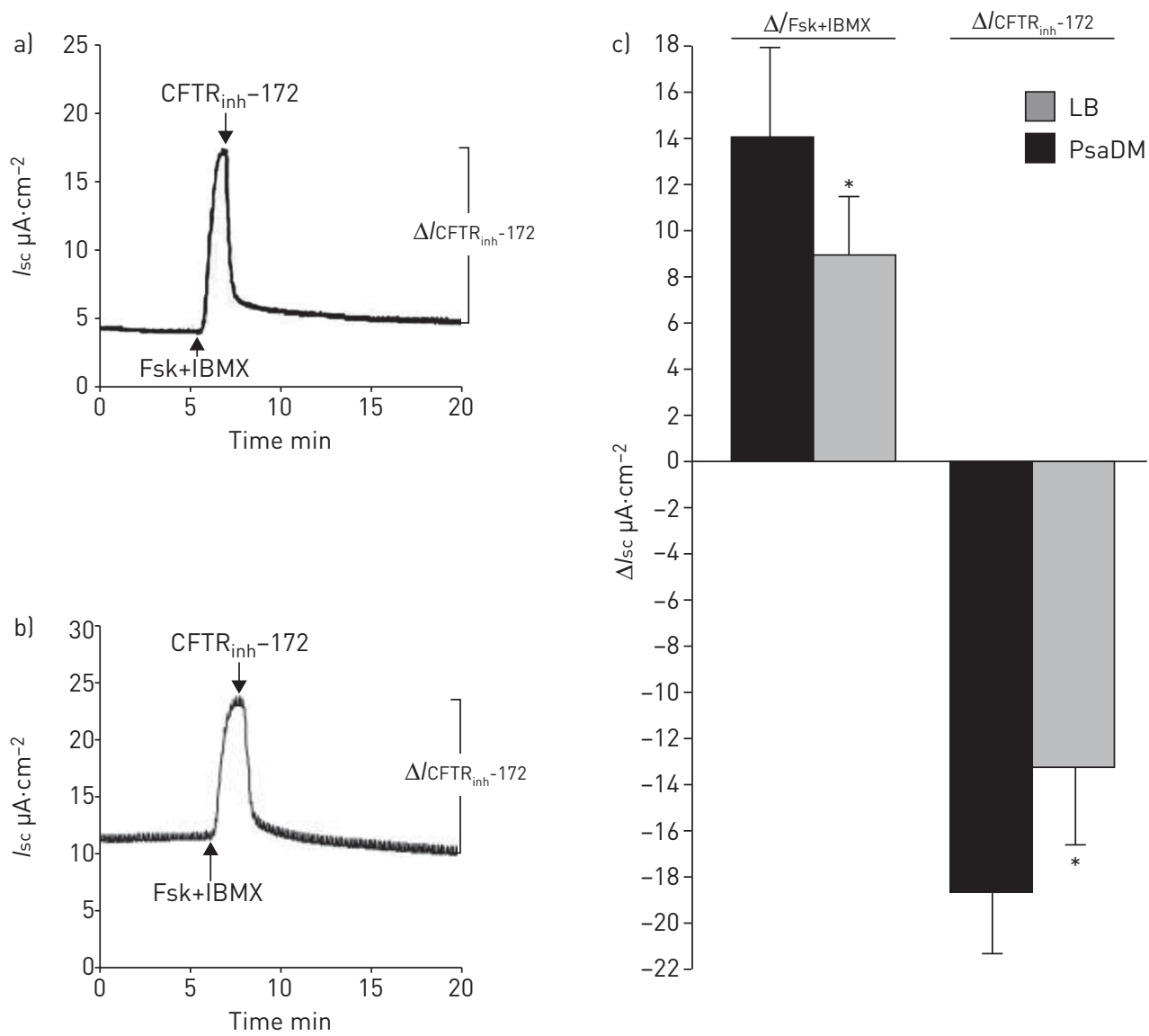

FIGURE 7 Inhibition of wildtype cystic fibrosis transmembrane conductance (wt-CFTR) function after Pseudomonas aeruginosa diffusible material (PsaDM) treatment in non-cystic fibrosis (CF) human primary airway cell monolayers. Short-circuit current $(I s c)$ measurements in an Ussing chamber were performed on non-CF human primary airway cell monolayers, cultured on permeant filters at the air-liquid interface, treated for $24 \mathrm{~h}$ with either $5 \%$ lysogeny broth (LB) medium or PsaDM. After mounting in an Ussing chamber, cells were bathed with an asymmetrical physiological solution (low chloride $\left(\mathrm{Cl}^{-}\right)$physiological solution at the apical side and high $\mathrm{Cl}^{-}$solution at the basolateral side), supplemented with $10 \mu \mathrm{M}$ amiloride. Upon Isc stabilisation, cells were treated with a combination of forskolin (Fsk) and 3-isobutyl-1-methylxanthine (IBMX), followed by $\mathrm{CFTR}_{\text {inh }}$-172. Representative Isc traces are reported for each condition a) LB and b) PsaDM while quantifications of the mean $\Delta I$ Fsk+IBMX and $\Delta I_{C_{F T R}} I_{\text {inh }}-172 I_{\text {sc }}$ are reported in c). $\mathrm{n}=7 ;{ }^{*}: \mathrm{p}<0.05$.

Similar to our results, SWIATECKA-URBan et al. [6] previously reported that a $4-6 \mathrm{~h}$ period of incubation with a culture of a $P$. aeruginosa laboratory isolate (PA14) reduced CFTR-mediated transepithelial $\mathrm{Cl}^{-}$secretion through Calu-3 cells as well as CFBE41o- cells stably expressing wt-CFTR. Importantly, we now confirmed the deleterious impact of a 24-h exposure to $P$. aeruginosa exoproducts in differentiated primary cultures of non-CF human airway cells (fig. 7). In contrast with these data, it has been shown that acute (15 min) application of $P$. aeruginosa (PA14) or purified LPS increased the rate of iodide efflux, an index of $\mathrm{Cl}^{-}$ secretion, through 2WT2 epithelial cells expressing wt-CFTR [9]. Furthermore, BuYCK et al. [35] have shown that $P$. aeruginosa LPS-stimulated $\mathrm{Cl}^{-}$currents through CFTR channels in 16HBE14o- cells, probably via a calcium signal. Similarly, N-(3-oxo-dodecanoyl)-S-homoserine lactone (3O-C12), a quorum sensing molecule secreted by $P$. aeruginosa, has been shown to rapidly enhance $\mathrm{Cl}^{-}$and fluid secretion as well as cytosolic calcium and cAMP levels [36]. It has also been reported that $P$. aeruginosa flagellin activated CFTR-dependent anion secretion in Calu-3 (through p38 signalling) as well as in primary cultures of human bronchial epithelial cells [37]. Most of these observations were interpreted as an attempt by airway epithelia to rapidly react to bacterial infection by increasing CFTR-dependent ion and fluid flow necessary for bacterial clearance from the airways. Notably, whereas pyocyanine (N-methyl-1-hydroxyphenazine) slightly activated basal $\mathrm{Cl}^{-}$secretion through CFBE-wt cells, this redox-active virulence factor produced by $P$. aeruginosa, severely inhibited Fsk-stimulated $\mathrm{Cl}^{-}$secretion [38]. Our results, as well as data from the literature, thus indicated that the effects of $P$. aeruginosa infection may be time- and model-dependent. Moreover, different $P$. aeruginosa products/virulence factors present in PsaDM could be responsible for the observed effects, and further studies would be necessary to clearly define their mechanisms of actions. 

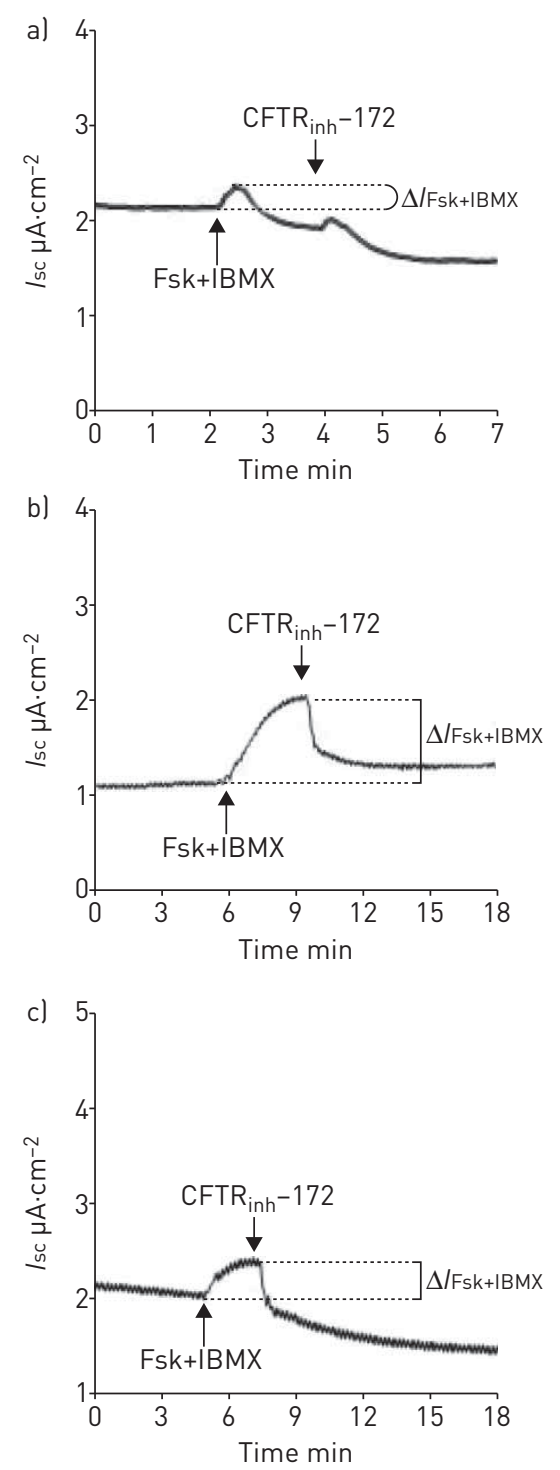

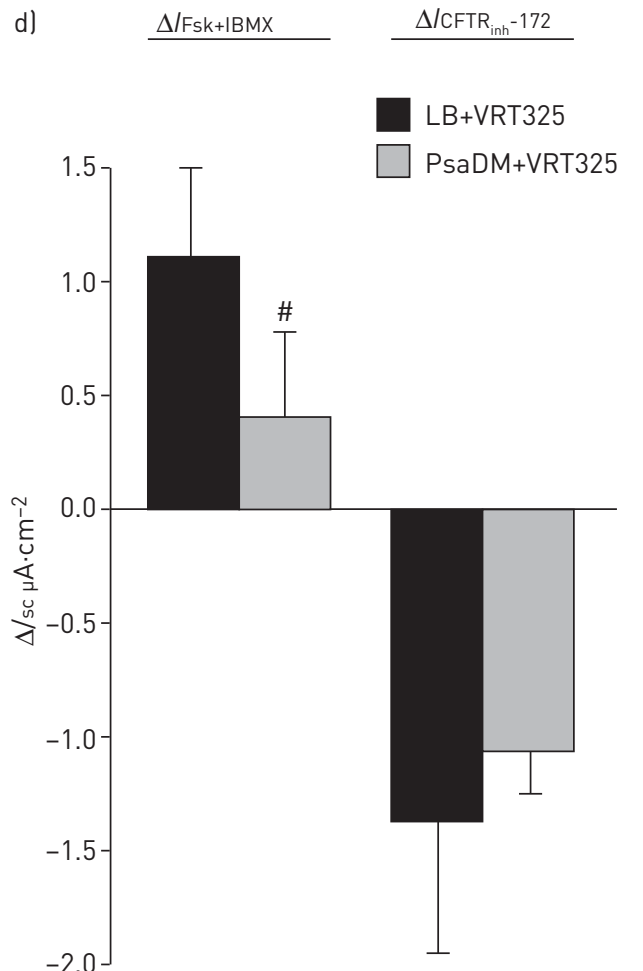

FIGURE 8 Inhibition of $\Delta$ F508 cystic fibrosis transmembrane conductance regulator ( $\Delta$ F508-CFTR) functional rescue after Pseudomonas aeruginosa diffusible material (PsaDM) treatment in cystic fibrosis (CF) human primary airway cell monolayers. Short-circuit current $\left(I_{s c}\right)$ measurements in an Ussing chamber were performed on CF human primary airway cell monolayers, cultured on permeant filters at the air-liquid interface and treated or not for $24 \mathrm{~h}$ with $10 \mu \mathrm{M}$ VRT-325 in combination with either 5\% lysogeny broth (LB) medium or PsaDM. After mounting in an Ussing chamber, filters were bathed with an asymmetrical physiological solution (low chloride ion $\left(\mathrm{Cl}^{-}\right)$physiological solution at the apical side and high $\mathrm{Cl}^{-}$at the basolateral side), supplemented with $10 \mu \mathrm{M}$ amiloride. Upon Isc stabilisation, the filters were treated with a combination of forskolin (Fsk) and 3-isobutyl-1-methylxanthine (IBMX), followed by CFTR $_{\text {inh }}$-172. Representative Isc traces are reported for each condition a) LB, b) LB+VRT-325, and c) PsaDM+VRT-325, while d) shows quantifications of the mean $\Delta I$ Fk+IBMX and $\Delta I_{C_{F T R}}$ inh $^{-172} I_{\text {sc. }} n=6 ;{ }^{*}: \mathrm{p}<0.03$.

\section{Impact of infection on CFTR expression}

In order to determine if the decrease in CFTR function after chronic exposure to $P$. aeruginosa products could be due to a decrease in CFTR expression, we then undertook PCR and immunoblot measurements to assess changes in CFTR mRNA and protein expression in CFBE-wt cells. Whereas the level of wt-CFTR mRNA was not affected after a 24-h treatment with PsaDM, we observed that both immature (band B) and mature (band C) CFTR proteins were severely reduced in CFBE-wt cells. In contrast with our results, a previous study [6] indicated that live P. aeruginosa bacteria (PA14 strain with 4-6 h of infection) had no effect on total cellular expression of wt-CFTR. However, it was shown that PA14 decreased the expression of wt-CFTR at the apical membrane, which is in agreement with the reduced function of CFTR observed under infectious conditions in that study as well as in ours. Moreover, our immunoblot assays after surface biotinylation as well as immunofluorescence analysis with confocal microscopy (fig. 3) showed reduced CFTR membrane expression after PsaDM exposure. 
It has to be noted that PACF508 used in our study is a clinical mucoid strain. However, it seems that the mucoid phenotype of $P$. aeruginosa is not responsible for the deleterious effect on CFTR since the non-mucoid laboratory strains PAO1 and PA14 have also been shown to downregulate CFTR $[6,39]$. The study by RUBino et al. [39] indicated that a mechanism involving bacterial pilin and flagellin as well as cellular NHERF1 could be involved in CFTR regulation by $P$. aeruginosa. In fact, various products secreted by $P$. aeruginosa have also been shown to have an impact on CFTR expression and function. Pyocyanin, for example, was found to inhibit CFTR expression at the apical membrane of airway epithelial cells [40]. The PA2394 protein (called CFTR inhibitory factor, Cif) secreted by P. aeruginosa is also involved in the reduction of CFTR apical membrane expression [41]. A model was then proposed where Cif secretion in $P$. aeruginosa outer membrane vesicles may reduce USP10-mediated deubiquitination of CFTR and secondarily increase the degradation of CFTR in lysosomes [42]. In agreement with CFTR degradation induced by infection, our results observed in the presence of $\mathrm{CHX}$ (fig. 4) indicated that the kinetics of wt-CFTR protein degradation could be slightly faster in the presence of PsaDM, compared to control conditions. However, this effect may not be sufficient to explain the huge inhibition of CFTR protein expression induced by PsaDM as shown in figure 2. In fact, our data obtained in the presence of MG132 and/or CHX (figs 4 and 5) indicated that CFTR synthesis may also be affected by PsaDM. Our study, as well as data from the literature, thus indicated that $P$. aeruginosa exoproducts may impact CFTR protein synthesis, degradation and trafficking/recycling to the cell membrane. A reduction in CFTR protein expression and function in non-CF airways exposed to chronic infection, for example in COPD patients, may have a negative impact on mucociliary clearance and consequently on the ability of airways to clear bacteria. This phenomenon could thus create a vicious circle of infection impairing CFTR, this CFTR dysfunction then favours infection.

\section{Impaired $\triangle F 508-C F T R$ rescue in the presence of infection}

Our immunoblot experiments on CFBE- $\Delta$ F508 cells first revealed that the immature band $\mathrm{B}$ of $\triangle$ F508-CFTR was decreased by a 24 -h exposure to PsaDM (fig. 2c-f). Furthermore, immunoblot and Ussing chamber measurements showed for the first time that $P$. aeruginosa exoproducts severely affected the ability of a CFTR corrector to rescue $\Delta$ F508-CFTR maturation and function in CFBE- $\Delta$ F508 cells as well as in CF primary airway epithelial cells (figs 6 and 8). These results are in agreement with a previous study [6] indicating that $P$. aeruginosa PA14 bacteria decreased CFTR currents induced by low temperature rescue in CFBE cells expressing $\triangle \mathrm{F} 508$-CFTR. The mechanisms whereby $\triangle \mathrm{F} 508$-CFTR rescue may be affected by $P$. aeruginosa remain to be elucidated.

Altogether, these data indicate that the presence of infection in CF patients may impair the ability of CFTR correctors to rescue the basic CFTR defect. These observations are critical to future screenings of CFTR correctors that should take into account the infectious component, in order to select small corrector molecules that could be efficient despite the presence of pathogens.

\section{Acknowledgements}

We acknowledge R.J. Bridges (Rosalind Franklin University, North Chicago, IL, USA) and Cystic Fibrosis Foundation Therapeutic Inc. (CFFT) (Bethesda, MA, USA) for providing VRT-325 as well as J. Riordan (University of North Carolina, Chapel Hill, NC, USA) and Cystic Fibrosis Foundation Therapeutic Inc for CFTR Ab596. Primary airway epithelial cells were from our Biobank of respiratory tissues of Centre de Recherche du Centre Hospitalier de l'Université de Montréal (CRCHUM), (Montreal, QC, Canada)/ Institut de Recherches Cliniques de Montréal (IRCM) of the Respiratory Health Network of FRQS. Finally, we acknowledge the logistical assistance from the Research Support Office, CRCHUM.

\section{References}

1 Boucher RC. Regulation of airway surface liquid volume by human airway epithelia. Pflugers Arch 2003; 445: 495-498.

2 Pezzulo AA, Tang XX, Hoegger MJ, et al. Reduced airway surface $\mathrm{pH}$ impairs bacterial killing in the porcine cystic fibrosis lung. Nature 2012; 487: 109-113.

3 Jacquot J, Tabary O, Le Rouzic P, et al. Airway epithelial cell inflammatory signalling in cystic fibrosis. Int $J$ Biochem Cell Biol 2008; 40: 1703-1715.

4 Eickmeier O, Huebner M, Herrmann E, et al. Sputum biomarker profiles in cystic fibrosis (CF) and chronic obstructive pulmonary disease (COPD) and association between pulmonary function. Cytokine 2010; 50: 152-157.

5 Cohen-Cymberknoh M, Kerem E, Ferkol T, et al. Airway inflammation in cystic fibrosis: molecular mechanisms and clinical implications. Thorax 2013; 68: 1157-1162.

6 Swiatecka-Urban A, Moreau-Marquis S, Maceachran DP, et al. Pseudomonas aeruginosa inhibits endocytic recycling of CFTR in polarized human airway epithelial cells. Am J Physiol Cell Physiol 2006; 290: C862-C872.

7 Zahm JM, Delavoie F, Toumi F, et al. Long acting $\beta 2$-agonist and corticosteroid restore airway glandular cell function altered by bacterial supernatant. Respir Res 2010; 11: 6 .

8 Le Gars M, Descamps D, Roussel D, et al. Neutrophil elastase degrades cystic fibrosis transmembrane conductance regulator via calpains and disables channel function in vitro and in vivo. Am J Respir Crit Care Med 2013; 187: 170-179. 
Haenisch MD, Ciche TA, Luckie DB. Pseudomonas or LPS exposure alters CFTR iodide efflux in 2WT2 epithelial cells with time and dose dependence. Biochem Biophys Res Commun 2010; 394: 1087-1092.

10 Donaldson SH, Boucher RC. Update on pathogenesis of cystic fibrosis lung disease. Curr Opin Pulm Med 2003; 9 : 486-491.

11 Folkesson A, Jelsbak L, Yang L, et al. Adaptation of Pseudomonas aeruginosa to the cystic fibrosis airway: an evolutionary perspective. Nat Rev Microbiol 2012; 10: 841-851.

12 Nichols DP, Konstan MW, Chmiel JF. Anti-inflammatory therapies for cystic fibrosis-related lung disease. Clin Rev Allergy Immunol 2008; 35: 135-153.

13 Ciofu O, Hansen CR, Hoiby N. Respiratory bacterial infections in cystic fibrosis. Curr Opin Pulm Med 2013; 19: 251-258.

14 Riordan JR. CFTR function and prospects for therapy. Annu Rev Biochem 2008; 77: 701-726.

15 Lubamba B, Dhooghe B, Noel S, et al. Cystic fibrosis: insight into CFTR pathophysiology and pharmacotherapy. Clin Biochem 2012; 45: 1132-1144.

16 Van Goor F, Straley KS, Cao D, et al. Rescue of $\triangle$ F508-CFTR trafficking and gating in human cystic fibrosis airway primary cultures by small molecules. Am J Physiol Lung Cell Mol Physiol 2006; 290: L1117-L1130.

17 Wang Y, Loo TW, Bartlett MC, et al. Additive effect of multiple pharmacological chaperones on maturation of CFTR processing mutants. Biochem J 2007; 406: 257-263.

18 Rowe SM, Pyle LC, Jurkevante A, et al. $\triangle$ F508 CFTR processing correction and activity in polarized airway and non-airway cell monolayers. Pulm Pharmacol Ther 2010; 23: 268-278.

19 Van Goor F, Hadida S, Grootenhuis PD, et al. Correction of the F508del-CFTR protein processing defect in vitro by the investigational drug VX-809. Proc Natl Acad Sci USA 2011; 108: 18843-18848.

20 Kim Chiaw P, Wellhauser L, Huan LJ, et al. A chemical corrector modifies the channel function of F508del-CFTR. Mol Pharmacol 2010; 78: 411-418.

21 Farinha CM, King-Underwood J, Sousa M, et al. Revertants, low temperature, and correctors reveal the mechanism of F508del-CFTR rescue by VX-809 and suggest multiple agents for full correction. Chem Biol 2013; 20: 943-955.

22 He L, Kota P, Aleksandrov AA, et al. Correctors of $\triangle$ F508 CFTR restore global conformational maturation without thermally stabilizing the mutant protein. FASEB J 2013; 27: 536-545.

23 Okiyoneda T, Veit G, Dekkers JF, et al. Mechanism-based corrector combination restores DeltaF508-CFTR folding and function. Nat Chem Biol 2013; 9: 444-454.

24 Becq F, Mettey Y, Gray MA, et al. Development of substituted Benzo[c]quinolizinium compounds as novel activators of the cystic fibrosis chloride channel. J Biol Chem 1999; 274: 27415-27125.

25 Robert R, Carlile GW, Pavel C, et al. Structural analog of sildenafil identified as a novel corrector of the F508del-CFTR trafficking defect. Mol Pharmacol 2008; 73: 478-489.

26 Kunzelmann K, Schwiebert EM, Zeitlin PL, et al. An immortalized cystic fibrosis tracheal epithelial cell line homozygous for the $\triangle$ F508 CFTR mutation. Am J Respir Cell Mol Biol 1993; 8: 522-529.

27 Bebok Z, Collawn JF, Wakefield J, et al. Failure of cAMP agonists to activate rescued $\triangle$ F508 CFTR in CFBE41oairway epithelial monolayers. J Physiol 2005; 569: 601-615.

28 Trinh NT, Bardou O, Privé A, et al. Improvement of defective cystic fibrosis airway epithelial wound repair after CFTR rescue. Eur Respir J 2012; 40: 1390-1400.

29 Berube J, Roussel L, Nattagh L, et al. Loss of cystic fibrosis transmembrane conductance regulator function enhances activation of p38 and ERK MAPKs, increasing interleukin-6 synthesis in airway epithelial cells exposed to Pseudomonas aeruginosa. J Biol Chem 2010; 285: 22299-22307.

30 Maille E, Trinh NT, Privé A, et al. Regulation of normal and cystic fibrosis airway epithelial repair processes by TNF- $\alpha$ after injury. Am J Physiol Lung Cell Mol Physiol 2011; 301: L945-L955.

31 Leroy C, Privé A, Bourret JC, et al. Regulation of ENaC and CFTR expression with $\mathrm{K}^{+}$channel modulators and effect on fluid absorption across alveolar epithelial cells. Am J Physiol Lung Cell Mol Physiol 2006; 291: L1207-L1219.

32 Leroy C, Dagenais A, Berthiaume Y, et al. Molecular identity and function in transepithelial transport of K(ATP) channels in alveolar epithelial cells. Am J Physiol Lung Cell Mol Physiol 2004; 286: L1027-L1037.

33 Trinh NT, Privé A, Maille E, et al. EGF and K+ channel activity control normal and cystic fibrosis bronchial epithelia repair. Am J Physiol Lung Cell Mol Physiol 2008; 295: L866-L880.

34 Trinh NT, Privé A, Kheir L, et al. Involvement of $\mathrm{K}_{\mathrm{ATP}}$ and KvLQT1 $\mathrm{K}^{+}$channels in EGF-stimulated alveolar epithelial cell repair processes. Am J Physiol Lung Cell Mol Physiol 2007; 293: L870-L882.

35 Buyck JM, Verriere V, Benmahdi R, et al. P. aeruginosa LPS stimulates calcium signaling and chloride secretion via CFTR in human bronchial epithelial cells. J Cyst Fibros 2013; 12: 60-67.

36 Schwarzer C, Wong S, Shi J, et al. Pseudomonas aeruginosa homoserine lactone activates store-operated cAMP and cystic fibrosis transmembrane regulator-dependent $\mathrm{Cl}^{-}$secretion by human airway epithelia. J Biol Chem 2010; 285: 34850-34863.

37 Illek B, Fu Z, Schwarzer C, et al. Flagellin-stimulated $\mathrm{Cl}^{-}$secretion and innate immune responses in airway epithelia: role for p38. Am J Physiol Lung Cell Mol Physiol 2008; 295: L531-L542.

38 Schwarzer C, Fischer H, Kim EJ, et al. Oxidative stress caused by pyocyanin impairs CFTR $\mathrm{Cl}^{-}$transport in human bronchial epithelial cells. Free Radic Biol Med 2008; 45: 1653-1662.

39 Rubino R, Bezzerri V, Favia $\mathrm{M}$, et al. Pseudomonas aeruginosa reduces the expression of CFTR via post-translational modification of NHERF1. Pflugers Arch 2014; 466: 2269-22788.

40 Kong F, Young L, Chen Y, et al. Pseudomonas aeruginosa pyocyanin inactivates lung epithelial vacuolar ATPase-dependent cystic fibrosis transmembrane conductance regulator expression and localization. Cell Microbiol 2006; 8: 1121-1133.

41 MacEachran DP, Ye S, Bomberger JM, et al. The Pseudomonas aeruginosa secreted protein PA2934 decreases apical membrane expression of the cystic fibrosis transmembrane conductance regulator. Infect Immun 2007; 75: 3902-3912.

42 Bomberger JM, Ye S, Maceachran DP, et al. A Pseudomonas aeruginosa toxin that hijacks the host ubiquitin proteolytic system. PLoS Pathog 2011; 7: e1001325. 